JOURNAL OF RESEARCH of the National Bureau of Standards - B. Mathematics and Mathematical Physics

Vol. 71B, No. 1, January-March 1967

\title{
The Coefficients of the Powers of a Polynomial
}

\author{
Morris Newman \\ Institute for Basic Standards, National Bureau of Standards, Washington, D.C. 20234
}

(December 5, 1966)

\begin{abstract}
It is shown that if $f(z)$ is a polynomial with no zeroes inside the unit circle and if $r$ is any positive number, then the coefficients of $f^{r}(z)$ tend to zero like $n^{-1-r}$, and this is best possible.

Key Words: Binomial coefficients, bounds, polynomials.
\end{abstract}

The general question which prompted this note is the following: Suppose that $f(z)$ is analytic and zero-free inside the unit circle, and normalized so that $f(0)=1$. Let $r$ be real and positive and choose (for definiteness) that determination of $f^{r}(z)$ for which $f^{r}(0)=1$. Then what can be said about the order of magnitude of the coefficients of $f^{r}(z)$ ? In particular, when will they converge to zero? In such a general setting no simple answer is to be expected and examples illustrating almost any sort of behavior may be given; but the case treated here, namely that when $f(z)$ is a polynomial, is reasonably simple. . In fact we shall prove the following theorem:

THEOREM 1. Let $\mathrm{f}(\mathrm{z})$ be a polynomial of degree $\mathrm{p}$ which is zero-free inside the unit circle and such that $\mathrm{f}(0)=1$. Let $\mathrm{r}$ be positive and suppose that

$$
\mathrm{f}^{\mathrm{r}}(\mathrm{z})=1+\sum_{\mathrm{n}=1}^{\infty} \mathrm{a}_{\mathrm{r}}(\mathrm{n}) \mathrm{z}^{\mathrm{n}}
$$

Then

$$
\mathrm{a}_{\mathrm{r}}(\mathrm{n})=0\left(\mathrm{n}^{-1-\mathrm{r}}\right), \quad \mathrm{n} \rightarrow \infty,
$$

and consequently

$$
\lim _{n \rightarrow \infty} a_{r}(n)=0 .
$$

The constant implied by (1) depends on $r$ and $p$, of course.

Precise inequalitieg rather than 0-estimates can be obtained at the expense of some additional complication, but we do not pursue this point here.

The statement is "best possible" in the sense that $a_{r}(n)$ is of true order of magnitude $n^{-1-r}$ for certain polynomials $f(z)$; for example for $f(z)=1+z$.
The statement is false for negative $r$. For example if $f(z)=(1-z)^{\mu}$ and $r=-2 / p$ then $a_{r}(n)=n+1$.

We require the following lemmas.

Lemma 1. Let $\mathrm{r}$ be arbitrary. Then

$$
\lim _{n \rightarrow \infty}(-1)^{n}\left(\begin{array}{l}
r \\
n
\end{array}\right) n^{1+r}=\frac{1}{\Gamma(-r)},
$$

and so

$$
\left(\begin{array}{l}
r \\
n
\end{array}\right)=0\left(n^{-1-r}\right), \quad n \rightarrow \infty
$$

Formula (2) is just Euler's definition of the $\Gamma$-function. Notice that if $r$ is a positive integer then $\left(\begin{array}{l}r \\ n\end{array}\right)$ vanishes for all sufficiently large $n$, which agrees with the fact that $\Gamma(s)$ has poles at $s=0,-1,-2, \ldots$.

Lemma 2. Let $\mathrm{r}$ be positive. For $\mathrm{p}=1,2,3$, . . define $\mathrm{t}_{\mathrm{p}}(\mathrm{n})$ by

$$
\sum_{n=0}^{\infty} t_{p}(n) z^{n}=\left\{1+\sum_{n=1}^{\infty} n^{-1-r} z^{n}\right\}^{p}
$$

Then

$$
t_{p}(n)=0\left(n^{-1-r}\right) .
$$

Proof. Differentiating (3) and comparing coefficients of corresponding powers of $z$ in the result we obtain the recurrence formula

$$
n t_{p}(n)=p \sum_{k=1}^{n} k^{-r} t_{p-1}(n-k) .
$$


The proof is by induction on $p$. For $p=1, t_{p}(n)=n^{-1-r}$, $n \geqslant 1$ and so the lemma is certainly true then. Assume the truth of the lemma for $p-1, p \geqslant 2$. Write (4) as

$$
\begin{aligned}
n t_{p}(n)=p & \sum_{1 \leqslant k \leqslant \frac{n-1}{2}} k^{-r} t_{p-1}(n-k) \\
+p & \sum_{\frac{n-1}{2}<k \leqslant n-1} k^{-r} t_{p-1}(n-k)+p n^{-r} .
\end{aligned}
$$

Then

$$
\begin{aligned}
\sum_{1 \leqslant k \leqslant \frac{n-1}{2}} k^{-r} t_{p-1}(n-k)=0 & \left\{\sum_{1 \leqslant k \leqslant \frac{n-1}{2}} k^{-r}(n-k)-1-r\right\} \\
=0 & \left\{n^{-1-r} \sum_{1 \leqslant k \leqslant \frac{n-1}{2}} k^{-r}\right\}=0\left(n^{-r}\right)
\end{aligned}
$$

(using the crude estimate $k^{r} \geqslant 1$ ), and

$$
\begin{array}{r}
\sum_{\frac{n-1}{2}<k \leqslant n-1} k^{-r} t_{p-1}(n-k)=0\left\{\sum_{\frac{n-1}{2}<k \leqslant n-1} k^{-r}(n-k)^{-1-r}\right\} \\
=0\left\{n^{-r} \sum_{\frac{n-1}{2}<k \leqslant n-1}(n-k)^{-1-r}\right\}=0\left(n^{-r}\right),
\end{array}
$$

since the series $\sum_{k=1}^{\infty} k^{-1-r}$ converges. Thus

$$
\begin{aligned}
& n t_{p}(n)=0\left(n^{-r}\right), \\
& t_{p}(n)=0\left(n^{-1-r}\right)
\end{aligned}
$$

and the lemma is proved for $p$. This concludes the proof of the lemma.

We turn now to the proof of theorem 1. We may write

$$
f(z)=\left(1+\alpha_{1} z\right)\left(1+\alpha_{2} z\right) \ldots\left(1+\alpha_{p} z\right),
$$

where, because of the assumptions made about $f(z)$, the numbers $\alpha_{i}$ are all of modulus not exceeding 1 .
We have

$$
\begin{aligned}
f^{r}(z) & =1+\sum_{n=1}^{\infty} a_{r}(n) z^{n} \\
& =\prod_{i=1}^{p}\left(1+\alpha_{i} z\right)^{r} \\
& =\prod_{i=1}^{p}\left\{\sum_{n i=0}^{\infty}\left(\begin{array}{c}
r \\
n
\end{array}\right) \alpha_{i}^{n} i z^{n} i\right\} \\
& \ll K\left\{1+\sum_{n=1}^{\infty} \frac{z^{n}}{n^{1+r}}\right\}^{p} \\
& =K \sum_{n=0}^{\infty} t_{p}(n) z^{n},
\end{aligned}
$$$$
=\prod_{i=1}^{p}\left\{\sum_{n i=0}^{\infty}\left(\begin{array}{c}
r \\
n i
\end{array}\right) \alpha_{i}^{n} i z^{n} i\right\} \ll\left\{\sum_{n=0}^{\infty}\left|\left(\begin{array}{c}
r \\
n
\end{array}\right)\right| z^{n}\right\}^{p}
$$

where $K$ is some suitable positive constant (lemma 1). ${ }^{1}$ Hence

$$
\left|a_{r}(n)\right| \leqslant K t_{p}(n),
$$

and lemma 2 implies the truth of the theorem for all positive $r$.

It is clear that the critical case is when $f(z)$ has all its roots on the unit circle, since otherwise much stronger inequalities for the coefficients $a_{r}(n)$ will hold. In fact, we can prove

TheOREM 2. Let $\mathrm{g}(\mathrm{z})$ be a polynomial of degree $\mathrm{p}$ such that $\mathrm{g}(0)=1$, and let $\mathrm{T}$ be the distance from the origin to the nearest zero of $\mathrm{g}(\mathrm{z})$. Let $\mathrm{r}$ be positive,

$$
g^{r}(z)=1+\sum_{n=1}^{\infty} b_{r}(n) z^{n}
$$

Then

$$
b_{r}(n)=0\left(n^{-1-r} T^{-n}\right), \quad n \rightarrow \infty .
$$

Proof. It is only necessary to apply theorem 1 to the polynomial $g(T z)=f(z)$ which now has no zeroes inside the unit circle and satisfies $f(0)=1$.

'We write $\sum a_{n} z^{n} \ll \sum b_{n} z^{n}$ to mean that $b_{n} \geqslant 0$ and $\left|a_{n}\right| \leqslant b_{n}$.

(Paper 71B1-190) 